\title{
Prospectively Recording Errors Is Valuable in Reducing Complications
}

\author{
Caroline Gunaratnam1, Adetunji Adeniyi Oremakinde², Mark Bernstein ${ }^{1}$ \\ ${ }^{1}$ Division of Neurosurgery and Joint Center for Bioethics, University Health Network, Toronto, Canada \\ ${ }^{2}$ Department of Neurological Surgery, University College Hospital, Ibadan, Nigeria \\ Email: caroline.gunaratnam@gmail.com
}

Received 5 August 2014; revised 20 September 2014; accepted 5 October 2014

Copyright (C) 2014 by authors and Scientific Research Publishing Inc.

This work is licensed under the Creative Commons Attribution International License (CC BY).

http://creativecommons.org/licenses/by/4.0/

(c) (i) Open Access

\begin{abstract}
Errors have traditionally been a less commonly discussed topic but are becoming increasingly examined due to a trend towards expanded awareness about the high human and financial cost of errors in medicine. Recording and reporting of errors has been a difficult issue in the health sector due to fear of litigation from patients, complaint to governing bodies, and embarrassment from colleagues. In this article we examine the advantages of prospective error recording. The studies on this subject report that the culture of prospective error recording is of high value in improving most parameters of errors and may indeed reduce complications.
\end{abstract}

Keywords

Prospective Study, Error Recording, Prevention, Neurosurgery, Prospective Value

\section{Introduction}

Errors are inevitable especially in complex systems such as the delivery of health care. Medical errors have enjoyed significant deliberation in the mass media [1] and the scientific articles [2]-[6]. Errors are common and are the cause of significant morbidity and mortality in the health sector [7] [8]. The 1999 Institute of Medicine Report showed that errors in the health sector were the eighth leading cause of death in the United States and account for up to 100,000 deaths annually [9]. One popularly cited retrospective study found that $66 \%$ of all adverse events were surgical and 54\% of the surgical adverse events were preventable [3]. Stone and Bernstein reported that of all the complications recorded in the neurosurgical practice of the senior author (MB), $16.7 \%$ were related to errors, of which $80.6 \%$ were major errors. Of the error related complications, $74.2 \%$ were declared preventable [7].

Errors can be defined as any act of omission or commission resulting in deviation from a perfect course for 
the patient; a perfect course can be defined as one in which nothing went wrong, from the smallest detail (such as dropping a sponge) to the most obvious example (one that every neurosurgeon would easily recognize) [8]. Errors may occur as a result of time constraints, physical and mental stress, communication problems, inattentiveness, negligence or lack of organization in health care teams or systems [8] [10]. Recording and reporting of errors and their complications by clinicians are essential, especially for neurosurgeons, whose complications can result in dramatic and catastrophic consequences for their patients [7] [11] [12]. It is a moral right and obligation for clinicians to openly recognize their fallibility and introduce strategies and methods for limiting the occurrence of medical errors, which might lead to complications and adverse events. It would also help to reduce the additional expenses and costs such as fines, legal settlements, and administration costs that the hospital and staff members must bear due to the occurrence of preventable errors [13]. The recognition and open discussion of errors may help facilitate a culture devoid of fear, shame, and recrimination of errors; however, this has not been traditionally encouraged or accepted despite the fact that the process of limiting errors is a necessary and integral component of quality assurance in the health sector. Most of the scientific literature is made up of retrospective studies. The disadvantages of retrospective error reporting, which may appear to some clinicians as a defensive and safe way to avoid troubles, are discussed in this article. The main focus of this review is the thorny issue of the value and benefit derived by clinicians and their patients from the practice of prospective error recording and reporting.

\section{Prospective vs. Retrospective Error Recording}

\subsection{Advantages of Retrospective Error Recording}

Retrospective error recording is a valid alternative method for clinicians who do not beleive in prospective error recording for one reason or the other. Many clinicians who do not want to actively and openly track their errors due to fear of litigation and criticism can still look back into their records, review the pattern of their errors, learn from the information, and formulate strategies to prevent errors. Suboptimal recording is clearly better than no recording. Therefore, this subset of clinicians can still benefit from retrospective recording; if retrospective recording is completely discouraged these subset of clinicians will be left with no other alternative method for recording errors.

\subsection{Disadvantages of Retrospective Error Recording}

The disadvantages of retrospective error recording include underreporting due to poor record keeping and the fact that some errors like near misses might not have been captured as they may not seem important to clinicians. In addition, retrospective studies work on the assumption that data will be found in the limited available records. The study design and procedures for collecting information on errors with retrospective error recording are far more inferior in comparison to prospective studies. Recording errors retrospectively would delay many benefits of error recording such as; delaying the time it takes for team members to realize the cause of preventable errors. It would also constitute delay and difficulty in detecting preventable errors that cause complications and adverse events. For example, it may be easy to detect a postoperative hemorrhage by checking the chart and the imaging record, but it will be next to impossible to determine how sleeplessness, distractions, poor communication, and technical factors contributed to the occurrence of the hemorrhage through a retrospective study [8]. Retrospective studies would not facilitate discussion among team members about errors committed after the surgery, nor would it facilitate discussion before surgery, about anticipated errors that should be prevented. There would also be much delay or time lag between the occurrence of an error and reporting it.

\subsection{Advantages of Prospective Error Recording}

Prospective error recording is a deliberate and well planned action in evaluating errors. This method is more accurate and its immediate effects of error recording such as, increased awareness and identification of error prone practices are likely to be more evident compared to information gained through retrospective recording. Etchells et al. noted that the rate of adverse events recorded in the largest retrospective study was approximately $10 \%$ while it is much higher in the prospective studies [8]. Rebesal et al. noted that prospective studies are more capable of picking up and identifying adverse events compared to retrospective studies at a ratio of approximately 6 to 1 [14]. They found that retrospective studies had adverse events in approximately 5\% of patients admitted 
while prospective studies published figures of approximately 30\% [14].

Prospective error recording makes it possible to establish the causal pathway for the occurrence of specific errors with accuracy, which could also play a significant role in providing preventive measures and changes in protocols to help improve the quality of care [14]-[16]. The actual process of documentation and recording of errors prospectively is important in order to gain access to real time occurrences as well as enhance the accuracy and details of each event [11] [12]. When errors are prospectively recorded it allows for the involvement of all team members and increases their sense of responsibility and communication not just with the patient, but with each other.

\subsection{Disadvantages of Prospective Error Recording}

Even though prospective recording of errors would allow health care systems and team members to benefit on a larger and smaller scale, it does come with a few disadvantages. With prospective error recoding, team members must always be well aware of their work environment, its recent changes and the kind of errors that these changes bring along. Team members, especially new staff must be well prepared to be able to adapt easily to these changes. The other disadvantage is that the clinicians who are afraid of litigation and other legal and professional implications of error recording will not find prospective error recording as convenient as retrospective recording. This is because prospective recording needs to be openly and actively done in conjunction with other health care workers.

\section{Value of Prospective Error Recording on Error Parameters}

A recent study by Oremakinde and Bernstein [11] showed the importance and impact of prospective error recording. The error types that were recorded prospectively include technical, contamination, nursing, anesthesia, management/judgment, and communication/information errors [11]. They found that, overtime these types of errors had significantly declined [11]. Two time epocs were compared. Group A involved error recorded in roughly the first half of a 13-year recording period which was previously published [7], while Group B involved the error recorded roughly in the last half of the study period [11]. Error parameters in Group A were compared with Group B. There was a significant decrease in the proportion of cases with error (87\% to $83 \%, p<0.006)$, mean errors per case (2.4 to $1.9, p<0.0001)$, proportion of error related complications $(16.7 \%$ to $5.5 \%, p<$ $0.002)$, and clinical impacts of error $(2.7 \%$ to $1.0 \%, p<0.0001)$ in Group B compared with Group A. Errors in Group B tended to be more preventable than those in Group A (85.8\% vs. $78.5 \%, p<0.0001)$. A descending trend in the mean errors per case was demonstrated over the whole study period. However, the severity of errors was higher in Group B compared to Group A. This is due to the prevalence of some near miss errors which are classified as severe errors because of their ability to cause serious adverse events if they are not intercepted.

The significant improvement of errors in Group B could be due to the continuous conversations about errors made in the neurosurgeons' (MB) practice, and the awareness of commonness and preventability of errors amongst all team members. There was also encouragement amongst team members to communicate with each other prior to and during each surgical procedure about specific details and peculiarities of each procedure, the instruments that would be needed, and the possible errors that could be committed and should be avoided. This was particularly prevalent amongst the trainees (i.e. residents and fellows) and nurses. The use of the surgical check list was also a very valuable tool that contributed to error reduction in Group B. These factors contribute to the Hawthorne effect, which is the positive subconscious change in behaviors and attitudes of workers when their actions are monitored in real time. This was also said to be a contributing factor to the reduction in error parameters in study Group B of the report by Oremakinde and Bernstein [11]. In another study, Rebesal et al. [14] reported that continuous prospective monitoring of adverse events has influence on the quality of care and the incidence of errors in general surgery. They found that the incidence of adverse events remained constant during the study period, but errors decreased from $11.1 \%$ to $4.5 \%(p=0.005)$ [14].

\section{Conclusion}

In this brief review we argue that prospective error recording is superior to retrospective error reporting. This is due to the reduction in underreporting of errors and improvement in the quality of patients' care, through early and continuous evaluation of preventive protocols on an ongoing basis. It may take time for the health care sys- 
tem and team members to adjust and adapt to this type of error recording, but it will encourage an attitude of collegial communication amongst health care team members and their patients about errors. It is also apparent that prospective error reporting may cause a strong attitudinal and behavioral change which includes the Hawthorne effect, transparency, disclosure, and communication of errors. This in turn might ultimately lead to the improvement in error prevention and reduction in error parameters and complications. What may seem new or strange today may hold to be the new norm in the future. Aiming to keep the occurrence of errors from very low to nil is key to having better surgical outcomes and patient experiences.

\section{References}

[1] Gawande, A. (1999) When Doctors Make Mistakes. The New Yorker, 1, 40-55.

[2] Brennan, T.A., Leape, L.L., Laird, N.M., et al. (1991) Incidence of Adverse Events and Negligence in Hospitalized Patients: Results of the Harvard Medical Practice Study I. New England Journal of Medicine, 324, 370-376. http://dx.doi.org/10.1056/NEJM199102073240604

[3] Gawande, A.A., Thomas, E.J., Zinner, M.J., et al. (1999) The Incidence and Nature of Surgical Adverse Events in Colorado and Utah in 1992. Surgery, 126, 66-75. http://dx.doi.org/10.1067/msy.1999.98664

[4] Leape, L.L., Brennan, T.A., Laird, N., et al. (1991) The Nature of Adverse Events in Hospitalized Patients: Results of the Harvard Medical Practice Study II. New England Journal of Medicine, 324, 277-384. http://dx.doi.org/10.1056/NEJM199102073240605

[5] Vincent, C., Neale, G. and Woloshynowych, M. (2001) Adverse Events in British Hospitals: Preliminary Retrospective Record Review. BMJ, 322, 517-519. http://dx.doi.org/10.1136/bmj.322.7285.517

[6] Wilson, R.M., Harrison, B.T., Gibberd, R.W., et al. (1999) An Analysis of the Causes of Adverse Events from the Quality of Australian Health Care Study. Medical Journal of Australia, 170, 411-415.

[7] Stone, S. and Bernstein, M. (2007) Prospective Error Recording in Surgery: An Analysis of 1108 Elective Neurosurgical Cases. Neurosurgery, 60, 1075-1082.

[8] Etchells, E., O’Neill, C. and Bernstein, M. (2003) Patient Safety in Surgery: Error Detection and Prevention. World Journal of Surgery, 27, 936-942. http://dx.doi.org/10.1007/s00268-003-7097-2

[9] Kohn, L.T., Corrigan, J.M. and Donaldson, M.S. (2000) Errors in Health Care: A Leading Cause of Death and Injury. In: To Err Is Human: Building a Sager Health System, National Academy Press, Washington DC, 26-48.

[10] Boström, J., Yacoub, A. and Schramm, J. (2010) Prospective Collection and Analysis of Error Data in a Neurosurgical Clinic. Clinical Neurology and Neurosurgery, 112, 314-319. http://dx.doi.org/10.1016/j.clineuro.2010.01.001

[11] Oremakinde, A.A. and Bernstein, M. (2014) A Reduction in Errors Is Associated with Prospectively Recording Them. Journal of Neurosurgery, 121, 297-304. http://dx.doi.org/10.3171/2014.5.JNS132341

[12] Bosma, E., Veen, E.J. and Roukema, J.A. (2011) Incidence, Nature and Impact of Error in Surgery. British Journal of Surgery, 98, 1654-1659. http://dx.doi.org/10.1002/bjs.7594

[13] Cohen, F.L., Mendelsohn, D. and Bernstein, M. (2010) Wrong-Site Craniotomy: Analysis of 35 Cases and Systems for Prevention. Journal of Neurosurgery, 113, 461-473. http://dx.doi.org/10.3171/2009.10.JNS091282

[14] Rebasa, P., Mora, L., Luna, A., Montmany, S., Vallverdú, H. and Havarro, S. (2009) Continuous Monitoring of Adverse Events: Influence on the Quality of Care and the Incidence of Errors in General Surgery. World Journal of Surgery, 33, 191-198. http://dx.doi.org/10.1007/s00268-008-9848-6

[15] Bernstein, M. (2003) Wrong-Side Surgery: Systems for Prevention. Canadian Journal of Surgery, 46, 144-146.

[16] Holliman, D. and Bernstein, M. (2012) Patients' Perception of Error during Craniotomy for Brain Tumor and Their Attitudes towards Pre-Operative Discussion of Error: A Qualitative Study. British Journal of Neurosurgery, 26, $236-330$. http://dx.doi.org/10.3109/02688697.2011.633642 
Scientific Research Publishing (SCIRP) is one of the largest Open Access journal publishers. It is currently publishing more than 200 open access, online, peer-reviewed journals covering a wide range of academic disciplines. SCIRP serves the worldwide academic communities and contributes to the progress and application of science with its publication.

Other selected journals from SCIRP are listed as below. Submit your manuscript to us via either submit@scirp.org or Online Submission Portal.
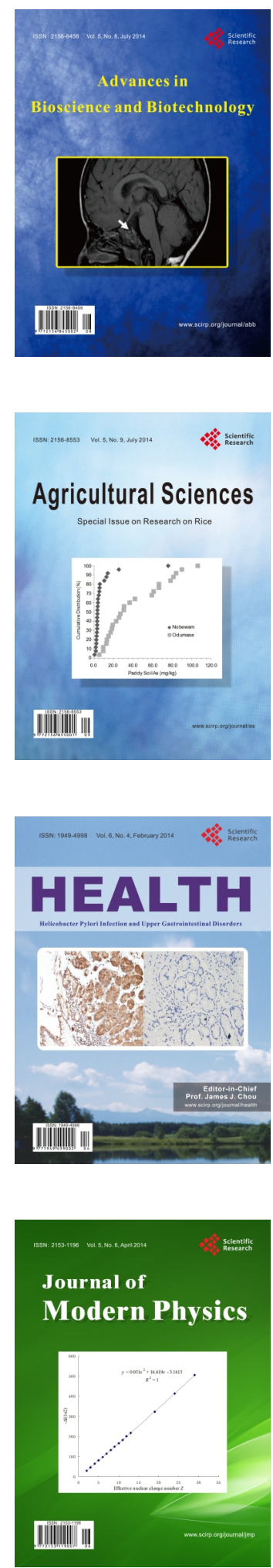
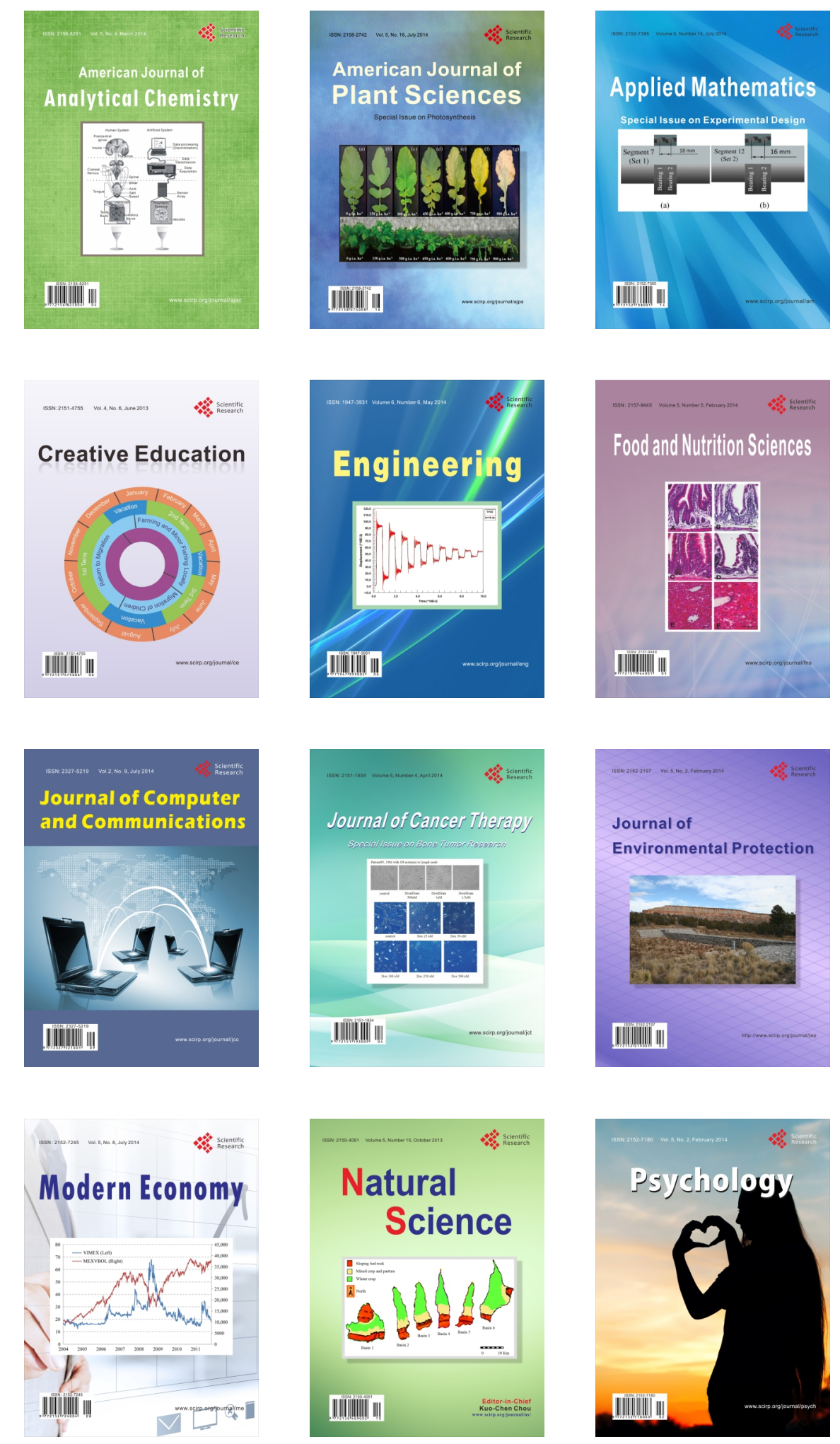